\title{
The Other Month Effect: A Reexamination of the "Other January" Anomaly
}

\author{
Ali F. Darrat \\ College of Business, Louisiana Tech University, Ruston, LA, 71272, USA
}

\section{Bin Li}

Griffith Business School, Griffith University, Nathan, QLD 4111, Australia

E-mail: b.li@griffith.edu.au

Tel: +61737357117

\section{Richard Chung}

Griffith Business School, Griffith University, Nathan, QLD 4111, Australia

Cooper, McConnell, and Ovtchinnikov (2006, CMO) find support for the "other January" effect in the US market over the period from January 1940 to December 2003 whereby the 11-month holding period returns following positive January returns are on average higher than those 11 months following negative January returns. Under this scenario, January returns can predict the subsequent 11-month holding period returns implying the potential for abnormal profits. We revisit this "anomaly" in the US stock market using the extended period from July 1926 to January 2012. Over the shorter period of 1940-2003 used by CMO, the results are supportive of the "other January" effect and they do so for several alternative holding periods. However, this alleged "other January" effect disappears once we expand the period. Moreover, we find similar and perhaps stronger anomalies for non-January months, particularly February and September. The evidence we uncover in this paper suggests that this alleged "other January" effect is likely sample-period sensitive and it is further not specific to the month of January.

Keywords: Seasonal anomalies; other January effect; market efficiency; January effect.

JEL Classifications: G14 


\section{Introduction}

The efficient market hypothesis that asset prices fully reflect all available information is at the core of finance theory (e.g., Fama, 1970). However, this key proposition has been exceedingly disputed in the empirical research through discovering anomalies and violations of the efficient market hypothesis.

Among the well-known anomalies are calendar effects such as the day-of-the-week effect, especially the Monday effect wherein Mondays display significantly negative average returns than other weekdays (Sun and Tong, 2002; Tu, 2003; Brusa and Liu, 2004; Gu, 2004). Another anomaly is the turn-of-the-month effect wherein stock returns are reportedly higher during the first few trading days of the month (Tonchev and Kim, 2004; Kunkel, Compton and Beyer, 2003). Research also reveals some patterns for a month-of-the-year effect, wherein returns in some months are significantly larger/lower than returns in other months. Most notably among these is the January effect that stock returns in January appear significantly greater than returns in other months of the year (Dzhabarov and Ziemba, 2010; Easterday, Sen and Stephan, 2009). Other studies report anomalies in other months particularly December (Russolillo, 2010; and Darrat, Li, Liu and Su, 2011).

We focus in this paper on another anomaly, the "other January" effect, recently discussed by Cooper, McConnell and Ovtchinnikov (2006). Their results over January 1940 to December 2003 suggest that the 11-month holding period returns following positive January returns are on average higher than those 11 months following negative January returns. Thus January returns can predict the subsequent 11-month holding period returns.

In a follow up study, Cooper, McConnell, and Ovtchinnikov (2009) investigate trading strategies using the "other January" effect, and report that the best strategy to earn excess return is to long stocks following a January positive stock returns, and invest in Treasury bills after a negative January stock return. Additional support for the "other January" effect comes from Sturm (2009) who finds that January returns have more predictive powers when the months are in the presidential election cycle.

However, other studies cast doubts on the "other January" effect. Marshall and Visaltanachoti (2010) argue that the trading strategy based on the "other January" effect is no better than a simple buy-and-hold strategy. Perhaps more importantly, several studies fail to find any support for the "other January" effect in international markets, suggesting that the "other January" effect is unique with the US market and cannot be generalized to other international markets ( see for example, Easton and Pinder, 2007; Stivers, Sun, and Sun, 2009; and Bohl and Salm, 2010).

Anomalies in stock returns should appeal to a wide range of market participants, such as portfolio managers and individual investors, in their search for when to buy or sell stocks. Knowledge on seasonality may also help listed companies and private firms as to the optimal timing for issuing new shares to effectively raise capital. If the anomalous pattern is fixed for a specific month, then informed investors can utilize this pattern to earn a risk-free profit. Therefore, investors could employ information on the anomaly findings to predict the future behavior of prices in violation to the efficient market hypothesis, at least in its weak-form sense. However, many alleged anomalies prove sample-period specific. Therefore it is important to use longer and updated time periods to check whether the alleged anomalies still 
exist. Several studies investigate the "other January" effect, but the research thus far has been limited to the 11-month holding period returns following the conditioning month. It seems useful to e examine the case for alternative holding periods.

We revisit the "other January" anomaly in the US stock market using the expanded period from July 1926 to January 2012. The start of our sample represents the earliest observations monthly stock returns available from Professor Kenneth French's Data Library (http://mba.tuck.dartmouth.edu/pages/faculty/ken.french/data_library.html). We also extend our analysis to holding period returns following other non-January months, which we term it as the "other month" effect. Furthermore, we examine the effect for the holding periods of 1 , 3, and 6 months, in addition to 11 months. Using the same sample period as Cooper, McConnell and Ovtchinnikov's (i.e., 1940-2003), the results confirm the "other January" effect for the alternative holding periods of 1, 3 and 6 months. However, this "anomaly" disappears once the sample is extended beyond 2003, suggesting that the "other January" effect is not only market specific, but it is also sample period specific. In addition, this effect may not be unique with January as similar results emerged with other months.

The rest of the paper is planned as follows. Section 2 describes the data and Section 3 outlines the methodology. Section 4 presents the empirical findings and Section 5 concludes.

\section{Data}

Our data are monthly and all come from French's Data Library covering the period from July 1926 through January 2012 (1,027 monthly observations). The excess return on the market is the value-weighted return on all NYSE, AMEX, and NASDAQ stocks (from the CRSP) minus the one-month Treasury bill rate (from Ibbotson Associates). We also convert the excess return to the raw return by adding it with the one-month Treasury bill rate. The return is shown in discrete terms. We calculate holding period returns by accumulating the period in each month over the number of holding period.

Table 1 provides summary statistics for the monthly excess market returns. The statistics include the number of observations, the numbers of positive and negative returns, mean, median, minimum, maximum, standard deviation, skewness, kurtosis, and the JarqueBera statistics for the monthly returns in different months. As the table suggests, the mean returns in September (-1.10\%) and October (-0.06\%) are much lower than the mean return in December (1.52\%) and January (1.27\%). December has the highest return and also generally displays the lowest standard deviation ${ }^{1}$. Generally speaking, the number of positive returns is greater than the number of negative returns except for September which has 39 positive returns and 46 negative returns. In unison with the mean/standard deviation statistics, December has the largest number of positive returns (68) and lowest number of negative returns (18). The Jarque-Bera statistics suggest that the monthly returns are not normally distributed except for January and November.

\footnotetext{
${ }^{1}$ Darrat, Li, Liu and Su (2011) report similar return characteristics for December in the US data.
} 
Table 1: Summary Statistics of Monthly Excess Stock Returns (July 1926 - January 2012)

\begin{tabular}{|c|c|c|c|c|c|c|c|c|c|c|c|c|}
\hline Variable & Number & $\begin{array}{c}\text { \# of } \\
\text { Positive } \\
\text { Returns }\end{array}$ & $\begin{array}{c}\text { \# of } \\
\text { Negative } \\
\text { Returns }\end{array}$ & Mean & Median & Std.Dev. & Min & Max & Skewness & Kurtosis & JB Statistics & JB p-value \\
\hline January & 86 & 54 & 32 & 1.27 & 1.49 & 4.74 & -7.93 & 13.58 & 0.26 & 0.04 & 0.98 & 0.61 \\
\hline February & 85 & 47 & 38 & 0.23 & 0.48 & 4.08 & -15.00 & 10.90 & -0.65 & 2.03 & 20.69 & 0.00 \\
\hline March & 85 & 53 & 32 & 0.42 & 0.93 & 4.93 & -23.70 & 8.80 & -1.84 & 6.58 & 201.25 & 0.00 \\
\hline April & 85 & 53 & 32 & 1.06 & 0.66 & 6.49 & -18.03 & 38.27 & 1.94 & 12.58 & 613.28 & 0.00 \\
\hline May & 85 & 52 & 33 & 0.23 & 1.34 & 5.81 & -22.01 & 21.15 & -0.86 & 4.88 & 94.75 & 0.00 \\
\hline June & 85 & 44 & 41 & 0.54 & 0.29 & 5.26 & -16.06 & 23.61 & 0.85 & 4.61 & 85.45 & 0.00 \\
\hline July & 86 & 47 & 39 & 1.21 & 1.19 & 5.90 & -10.83 & 33.72 & 1.76 & 9.60 & 374.43 & 0.00 \\
\hline August & 86 & 53 & 33 & 0.95 & 1.17 & 6.09 & -16.20 & 36.57 & 2.07 & 13.39 & 704.21 & 0.00 \\
\hline September & 86 & 39 & 46 & -1.10 & -0.34 & 5.97 & -29.04 & 15.95 & -1.25 & 5.05 & 114.08 & 0.00 \\
\hline October & 86 & 47 & 39 & -0.06 & 0.54 & 6.31 & -23.14 & 16.05 & -1.06 & 2.97 & 47.87 & 0.00 \\
\hline November & 86 & 58 & 28 & 1.18 & 1.88 & 5.22 & -12.70 & 11.75 & -0.61 & 0.28 & 5.66 & 0.06 \\
\hline December & 86 & 68 & 18 & 1.52 & 1.52 & 3.67 & -13.46 & 10.30 & -0.89 & 2.73 & 37.94 & 0.00 \\
\hline ALL & 1027 & 615 & 411 & 0.62 & 0.96 & 5.46 & -29.04 & 38.27 & 0.17 & 7.44 & 2370.40 & 0.00 \\
\hline
\end{tabular}

Notes: All returns are in percentages. 


\section{Methodology}

To test the mean differences, we use a simple t-test. We examine the other month effect by comparing the difference between the average of the accumulated k-month returns following the positive conditioning month and the average of the accumulated k-month returns following the negative conditioning month. The conditioning months range from January to December. For example, if it is January, we are testing the other January effect. If December, we are testing the other December effect, etc. The holding period $\mathrm{K}$ ranges from $1,3,6$, and 11 months.

The means test is essentially the same as the linear regression used in Cooper, McConnell and Ovtchinnikov (2006), where the accumulated 11 months returns are regressed on a dummy variable taking one when the January return is positive and zero otherwise ${ }^{2}$.

\section{Empirical Results}

Table 2 presents the results for our expanded sample period over July 1926 through January 2012. We report the mean and the standard deviation of the accumulated k-month excess market returns following any positive/negative return in any of the 12 conditioning months. We also provide the number of positive and negative conditioning months. The holding period $\mathrm{k}$ varies over $1,3,6$, and 11. Furthermore, to test the other month effect, we provide the spread between the accumulated k-month excess market returns following positive and those following the negative returns along with their associated standard errors.

Consider first the "other January" effect of Cooper, McConnell and Ovtchinnikov (2006) within our expanded period (July 1926 - January 2012). Contrary to them, the results in Table 2 indicate that the 11-month returns following positive January returns are not statistically different from those following negative January returns. Rather, the 6-month accumulated returns following positive January returns are on average 5.30\% larger than those following negative January returns. This finding suggests that there is a momentum in the first 6 months, but it reverts in the following 5 months.

Second, another interesting feature of Table 2 is that holding period returns following positive February returns are lower than those following negative February returns. For example, the spread for 3-month holding period is $-6.50 \%$ and the spread for the 6-month holding period is $-7.21 \%$, and both are statistically significant at the $5 \%$ level.

Third, though September has less positive than negative returns (see Table 1), the 3 month and 6-month holding period returns have higher average returns following positive September returns. For other months, there are no statistical difference between the returns following positive returns in that conditioning month and the ones following negative returns.

We check the robustness of our results, as Cooper, McConnell and Ovtchinnikov (2006) did, by performing the tests on raw market returns. As Table 3 suggests, the results remain essentially the same as those reported in Table 2 .

\footnotetext{
${ }^{2}$ Cooper, McConnell and Ovtchinnikov also use a randomized-bootstrap procedure but the results are essentially the same as those from the simple mean test. To minimize unnecessary econometrical complexity, we confine our attention to results from simple means tests.
} 
Table 2: Testing “Other Month" Anomalies (July 1926 - January 2012)

\begin{tabular}{|c|c|c|c|c|c|c|c|c|c|c|c|c|}
\hline & \multicolumn{3}{|c|}{1} & \multicolumn{3}{|c|}{3} & \multicolumn{3}{|c|}{6} & \multicolumn{3}{|c|}{11} \\
\hline & + & - & Diff & + & - & Diff & + & - & Diff & + & - & Diff \\
\hline \multirow[t]{3}{*}{ January } & 0.75 & -0.62 & 1.37 & 2.70 & -0.27 & $2.96^{*}$ & 5.70 & -0.14 & $5.83^{* *}$ & 8.90 & 2.66 & 6.24 \\
\hline & (4.29) & (3.61) & $(0.85)$ & $(6.94)$ & (7.67) & (1.65) & (10.76) & (12.85) & (2.68) & (18.94) & (20.75) & (4.46) \\
\hline & [53] & [32] & & [53] & [32] & & [53] & [32] & & [53] & [32] & \\
\hline \multirow[t]{3}{*}{ February } & 0.30 & 0.57 & -0.27 & -0.43 & 4.95 & $-5.37 *$ & 1.95 & 7.52 & -5.57 & 6.09 & 10.46 & -4.37 \\
\hline & (5.27) & $(4.55)$ & (1.06) & (10.50) & (14.64) & $(2.84)$ & $(9.20)$ & (20.51) & (3.53) & (17.81) & (27.43) & $(5.05)$ \\
\hline & [47] & [38] & & [47] & [38] & & [47] & [38] & & [47] & [38] & \\
\hline \multirow[t]{3}{*}{ March } & 1.94 & -0.38 & 2.32 & 3.59 & -0.27 & 3.86 & 3.65 & 2.21 & 1.44 & 8.65 & 7.09 & 1.56 \\
\hline & $(6.41)$ & (6.47) & (1.45) & (14.23) & (13.06) & (3.02) & (15.75) & (18.37) & (3.82) & (21.47) & (26.43) & (5.48) \\
\hline & [53] & [32] & & [53] & [32] & & [53] & [32] & & [52] & [32] & \\
\hline \multirow[t]{3}{*}{ April } & 0.81 & -0.74 & 1.55 & 2.45 & 0.88 & 1.57 & 3.19 & -1.11 & 4.30 & 9.80 & 2.92 & 6.89 \\
\hline & (5.56) & (6.17) & (1.36) & $(8.66)$ & (8.78) & (1.94) & (12.27) & (14.95) & (3.06) & (18.66) & (23.78) & (4.87) \\
\hline & [53] & [32] & & [53] & [32] & & [53] & [32] & & [52] & [32] & \\
\hline \multirow[t]{3}{*}{ May } & 0.28 & 0.96 & -0.68 & 1.88 & 4.23 & -2.35 & 3.67 & 1.34 & 2.32 & 8.54 & 7.30 & 1.24 \\
\hline & (3.85) & $(6.97)$ & (1.29) & (6.99) & (17.26) & (3.10) & (13.40) & $(16.80)$ & (3.41) & (18.74) & (25.33) & (5.09) \\
\hline & [52] & [33] & & [52] & [33] & & [52] & [33] & & [52] & [32] & \\
\hline \multirow[t]{3}{*}{ June } & 0.49 & 1.94 & -1.45 & 1.01 & 1.42 & -0.41 & 4.47 & 3.41 & 1.06 & 8.64 & 7.63 & 1.00 \\
\hline & (4.70) & (7.01) & (1.31) & $(8.60)$ & (16.55) & $(2.90)$ & (13.95) & (17.44) & (3.44) & (20.19) & (28.68) & (5.45) \\
\hline & [44] & [41] & & [44] & [41] & & [44] & [41] & & [44] & [40] & \\
\hline \multirow[t]{3}{*}{ July } & 1.43 & 0.37 & 1.06 & 0.33 & -1.13 & 1.46 & 4.83 & 3.01 & 1.82 & 8.70 & 5.48 & 3.22 \\
\hline & $(6.21)$ & (5.96) & (1.28) & $(9.23)$ & $(9.80)$ & (2.03) & (14.44) & (14.63) & (3.09) & (22.20) & (22.53) & (4.84) \\
\hline & [47] & [39] & & [47] & [39] & & [47] & [39] & & [47] & [38] & \\
\hline \multirow[t]{3}{*}{ August } & -1.09 & -1.12 & 0.04 & -0.45 & 1.16 & -1.61 & 2.98 & 4.03 & -1.05 & 6.93 & 6.92 & 0.02 \\
\hline & (5.81) & $(6.31)$ & (1.34) & (12.64) & (8.59) & $(2.31)$ & (15.94) & (13.13) & (3.21) & (20.59) & (17.90) & (4.24) \\
\hline & [53] & [33] & & [53] & [33] & & [53] & [32] & & [53] & [32] & \\
\hline \multirow[t]{3}{*}{ September } & 1.22 & -1.13 & $2.35^{*}$ & 5.76 & 0.22 & $5.53 * *$ & 8.47 & 1.75 & $6.72 * *$ & 10.53 & 7.23 & 3.30 \\
\hline & (3.77) & (7.70) & (1.27) & (6.67) & (10.93) & (1.91) & (11.61) & (16.12) & (3.01) & (18.17) & (18.93) & $(4.01)$ \\
\hline & [39] & [47] & & [39] & [47] & & [39] & [46] & & [39] & [46] & \\
\hline \multirow[t]{3}{*}{ October } & 1.51 & 0.79 & 0.73 & 3.96 & 4.22 & -0.27 & 5.32 & 6.73 & -1.41 & 6.83 & 9.27 & -2.45 \\
\hline & (4.35) & (6.15) & (1.16) & (8.01) & (9.02) & (1.83) & (13.08) & (13.10) & (2.82) & (17.34) & $(21.20)$ & (4.21) \\
\hline & [47] & [39] & & [47] & [39] & & [46] & [39] & & [46] & [39] & \\
\hline \multirow[t]{3}{*}{ November } & 1.77 & 1.01 & 0.76 & 3.65 & 1.78 & 1.87 & 6.50 & 1.94 & 4.56 & 7.55 & 3.40 & 4.15 \\
\hline & (2.98) & (4.83) & $(0.98)$ & (7.39) & (8.65) & (1.91) & (10.40) & (19.90) & (3.94) & (15.53) & (21.39) & (4.47) \\
\hline & [58] & [28] & & [58] & [27] & & [58] & [27] & & [58] & [27] & \\
\hline \multirow[t]{3}{*}{ December } & 1.22 & 1.49 & -0.27 & 2.32 & 0.38 & 1.94 & 4.92 & 0.18 & 4.74 & 6.88 & 2.96 & 3.92 \\
\hline & (4.20) & (6.54) & (1.61) & (7.37) & (11.38) & (2.77) & (12.32) & (20.65) & (5.08) & (17.48) & $(24.70)$ & (6.14) \\
\hline & {$[68]$} & {$[18]$} & & [67] & [18] & & [67] & [18] & & [67] & [18] & \\
\hline
\end{tabular}

Notes: All returns are in percentages. 1, 3, 6, and 11 indicate holding periods. "Diff" refers to the spread between returns following positive returns in the conditioning month and those following negative returns. Standard errors are placed in parentheses below the means. Numbers of positive/negative returns are placed in squared brackets. ** and * denote statistical significance at the $5 \%$ and $10 \%$ levels, respectively. 
Table 3: Testing the "Other Month" Anomalies with Raw Returns (July 1926 - January 2012)

\begin{tabular}{|c|c|c|c|c|c|c|c|c|c|c|c|c|}
\hline & \multicolumn{3}{|c|}{1} & \multicolumn{3}{|c|}{3} & \multicolumn{3}{|c|}{6} & \multicolumn{3}{|c|}{11} \\
\hline & + & - & Diff & + & - & Diff & + & - & Diff & + & - & Diff \\
\hline \multirow[t]{3}{*}{ January } & 1.06 & -0.50 & $1.56^{*}$ & 3.38 & 0.76 & 2.62 & 7.08 & 1.99 & $5.08^{* *}$ & 11.68 & 6.54 & 5.14 \\
\hline & $(4.25)$ & (3.59) & $(0.86)$ & $(7.01)$ & (7.63) & (1.66) & (11.32) & (11.75) & $(2.58)$ & (19.66) & (18.87) & $(4.25)$ \\
\hline & [55] & [30] & & [55] & [30] & & [55] & [30] & & [55] & [30] & \\
\hline \multirow{2}{*}{ February } & (5.54) & (3.96) & (1.03) & (10.59) & (14.47) & (2.84) & (10.02) & (19.83) & (3.52) & (17.89) & (27.10) & (5.05) \\
\hline & [49] & [36] & & [49] & [36] & & [49] & [36] & & [49] & [36] & \\
\hline \multirow[t]{3}{*}{ March } & 2.23 & -0.08 & 2.31 & 4.50 & 0.61 & 3.88 & 5.50 & 3.94 & 1.56 & 12.10 & 10.27 & 1.83 \\
\hline & (6.39) & (6.49) & (1.45) & (14.16) & (13.02) & (3.01) & (15.51) & (18.16) & (3.77) & (20.81) & (26.54) & (5.44) \\
\hline & [53] & [32] & & [53] & [32] & & [53] & [32] & & [52] & [32] & \\
\hline \multirow{2}{*}{ April } & $(5.53)$ & (6.27) & (1.39) & (8.47) & (8.74) & (1.95) & (11.93) & (14.92) & (3.10) & (18.03) & (24.15) & (4.98) \\
\hline & [55] & [30] & & [55] & [30] & & [55] & [30] & & [54] & [30] & \\
\hline \multirow[t]{3}{*}{ May } & 0.64 & 1.15 & -0.51 & 2.71 & 5.30 & -2.59 & 5.39 & 3.17 & 2.21 & 11.91 & 10.55 & 1.36 \\
\hline & $(3.82)$ & (7.02) & (1.31) & (6.72) & (17.39) & (3.15) & (12.97) & (17.01) & (3.44) & (17.94) & $(25.90)$ & (5.17) \\
\hline & [53] & [32] & & [53] & [32] & & [53] & [32] & & [53] & [31] & \\
\hline \multirow[t]{3}{*}{ June } & 0.71 & 2.40 & -1.69 & 1.69 & 2.59 & -0.91 & 6.08 & 5.38 & 0.70 & 11.63 & 11.35 & 0.28 \\
\hline & (4.63) & (7.03) & $(1.32)$ & (8.42) & (16.79) & (2.97) & (13.39) & (17.76) & (3.45) & (19.58) & $(29.20)$ & $(5.52)$ \\
\hline & [46] & [39] & & [46] & [39] & & [46] & [39] & & [46] & [38] & \\
\hline \multirow[t]{2}{*}{ July } & 1.67 & 0.73 & 0.93 & 1.07 & -0.03 & 1.11 & 6.33 & 5.15 & 1.19 & 11.54 & 9.42 & 2.12 \\
\hline & $(6.22)$ & (5.92) & $(1.28)$ & $(9.08)$ & $(9.91)$ & (2.03) & (14.25) & (14.80) & (3.10) & (21.84) & (22.81) & $(4.86)$ \\
\hline \multirow[t]{3}{*}{ August } & -0.77 & -0.86 & 0.09 & 0.42 & 2.14 & -1.72 & 4.49 & 6.32 & -1.83 & 9.77 & 11.12 & -1.35 \\
\hline & (5.79) & (6.36) & (1.36) & (12.51) & (8.75) & $(2.32)$ & (15.80) & (13.19) & $(3.21)$ & (20.38) & $(17.70)$ & $(4.20)$ \\
\hline & [54] & [32] & & [54] & [32] & & [54] & [31] & & [54] & [31] & \\
\hline \multirow[t]{3}{*}{ September } & 1.12 & -0.67 & 1.79 & 6.10 & 1.03 & $5.07 * *$ & 9.27 & 3.76 & $5.51 *$ & 14.18 & 9.80 & 4.38 \\
\hline & (3.82) & (8.07) & (1.36) & $(6.26)$ & (11.62) & $(2.02)$ & (11.13) & $(17.21)$ & (3.16) & (16.77) & (19.93) & (3.98) \\
\hline & [44] & [42] & & [44] & [42] & & [44] & [41] & & [44] & [41] & \\
\hline \multirow[t]{3}{*}{ October } & 1.98 & 0.82 & 1.16 & 5.10 & 4.76 & 0.34 & 7.50 & 8.05 & -0.55 & 10.62 & 12.05 & -1.43 \\
\hline & $(4.55)$ & (6.01) & (1.16) & (8.11) & (8.92) & (1.83) & (13.25) & (12.92) & $(2.81)$ & $(17.29)$ & (20.98) & (4.19) \\
\hline & [48] & [38] & & [48] & [38] & & [47] & [38] & & [47] & [38] & \\
\hline \multirow[t]{3}{*}{ November } & 2.07 & 1.27 & 0.80 & 4.53 & 2.58 & 1.95 & 8.20 & 3.73 & 4.47 & 10.79 & 6.80 & 3.99 \\
\hline & $(2.86)$ & (4.93) & (1.01) & (7.10) & (9.10) & (1.99) & (10.02) & (20.47) & $(4.10)$ & (14.94) & (21.55) & (4.54) \\
\hline & [59] & [27] & & [59] & [26] & & [59] & [26] & & [59] & [26] & \\
\hline \multirow[t]{3}{*}{ December } & 1.46 & 2.01 & -0.54 & 3.16 & 1.12 & 2.04 & 6.35 & 2.86 & 3.49 & 10.14 & 5.99 & 4.15 \\
\hline & $(4.23)$ & $(6.72)$ & (1.73) & (7.38) & (11.87) & (3.02) & (12.81) & (20.17) & (5.24) & (17.79) & (23.67) & $(6.22)$ \\
\hline & [70] & [16] & & [69] & [16] & & [69] & [16] & & [69] & [16] & \\
\hline
\end{tabular}

Notes: See notes to Table 2. 
Table 4: Testing the "Other Month" Anomalies with Alternative Holding Periods (1940 January - December 2003)

\begin{tabular}{|c|c|c|c|c|c|c|c|c|c|c|c|c|}
\hline & \multicolumn{3}{|c|}{1} & \multicolumn{3}{|c|}{3} & \multicolumn{3}{|c|}{6} & \multicolumn{3}{|c|}{11} \\
\hline & + & - & Diff & + & - & Diff & + & - & Diff & + & - & Diff \\
\hline \multirow[t]{3}{*}{ January } & 0.77 & -0.99 & $1.75 * *$ & 3.27 & -0.66 & $3.93^{* *}$ & 6.47 & -2.69 & $9.16^{\text {*** }}$ & 12.25 & -2.98 & $15.22 * *$ \\
\hline & (3.69) & (3.03) & $(0.83)$ & (6.50) & $(6.01)$ & $(1.59)$ & (7.69) & (11.85) & $(2.68)$ & (12.84) & (14.89) & (3.59) \\
\hline & [41] & [23] & & [41] & [23] & & [41] & [23] & & [41] & [23] & \\
\hline \multirow{2}{*}{ February } & $(2.57)$ & (4.60) & $(0.94)$ & (7.59) & (8.48) & $(2.00)$ & (8.49) & (12.37) & $(2.70)$ & (13.14) & (17.34) & (3.86) \\
\hline & [35] & [29] & & [35] & [29] & & [35] & [29] & & [35] & [29] & \\
\hline \multirow[t]{3}{*}{ March } & 1.23 & -0.05 & 1.28 & 2.73 & -0.48 & 3.21 & 2.24 & 0.77 & 1.47 & 6.04 & 10.09 & -4.06 \\
\hline & $(3.72)$ & (4.85) & (1.17) & (6.75) & (9.93) & (2.31) & (11.02) & (14.63) & (3.48) & (16.43) & (17.37) & $(4.42)$ \\
\hline & [42] & [22] & & [42] & [22] & & [42] & [22] & & [42] & [22] & \\
\hline \multirow{2}{*}{ April } & $(4.86)$ & (4.49) & (1.19) & (7.00) & (8.06) & (1.98) & (9.71) & (11.34) & (2.76) & (14.72) & (20.36) & $(4.73)$ \\
\hline & [41] & [23] & & [41] & [23] & & [41] & [23] & & [41] & [23] & \\
\hline \multirow[t]{3}{*}{ May } & 0.31 & 0.47 & -0.16 & 1.19 & 1.19 & 0.01 & 2.36 & 2.41 & -0.04 & 7.20 & 9.31 & -2.10 \\
\hline & $(3.20)$ & (4.44) & (1.03) & (6.55) & (8.81) & (2.06) & (11.41) & $(10.40)$ & (2.76) & (17.76) & (15.35) & (4.19) \\
\hline & [41] & [23] & & [41] & [23] & & [41] & [23] & & [41] & [23] & \\
\hline \multirow[t]{3}{*}{ June } & 0.66 & 0.26 & 0.40 & 1.63 & -1.74 & 3.37 & 5.38 & 2.15 & 3.23 & 11.11 & 5.45 & 5.65 \\
\hline & $(3.81)$ & (4.59) & (1.05) & (6.74) & (10.90) & $(2.28)$ & (10.53) & (12.80) & (2.93) & (16.78) & (19.22) & (4.47) \\
\hline & [35] & [29] & & [35] & [29] & & [35] & [29] & & [35] & [29] & \\
\hline \multirow[t]{2}{*}{ July } & 0.70 & -0.06 & 0.76 & 0.14 & -0.13 & 0.28 & 4.80 & 4.93 & -0.13 & 8.61 & 8.15 & 0.46 \\
\hline & $(2.97)$ & (6.14) & (1.19) & (7.33) & (8.69) & (1.96) & (11.76) & (11.74) & (2.86) & (16.57) & (19.91) & (4.48) \\
\hline \multirow[t]{3}{*}{ August } & -0.28 & -1.55 & 1.27 & 1.27 & 1.14 & 0.13 & 5.51 & 3.68 & 1.83 & 8.94 & 7.62 & 1.32 \\
\hline & (3.68) & (5.02) & (1.12) & $(9.31)$ & (6.77) & (2.02) & (11.87) & (11.87) & (3.01) & (16.86) & $(18.45)$ & (4.47) \\
\hline & [37] & [27] & & [37] & [27] & & [37] & [27] & & [37] & [27] & \\
\hline \multirow[t]{3}{*}{ September } & 1.03 & 0.20 & 0.82 & 5.24 & 2.93 & 2.31 & 8.39 & 5.52 & 2.87 & 10.73 & 8.96 & 1.77 \\
\hline & (3.63) & $(6.22)$ & $(1.22)$ & $(6.91)$ & (7.98) & $(1.82)$ & (12.07) & (13.52) & (3.19) & (19.25) & (16.98) & $(4.61)$ \\
\hline & [26] & [38] & & [26] & [38] & & [26] & [38] & & [26] & [38] & \\
\hline \multirow[t]{3}{*}{ October } & 1.63 & 1.31 & 0.32 & 4.27 & 5.46 & -1.19 & 6.84 & 6.95 & -0.11 & 7.80 & 8.96 & -1.16 \\
\hline & $(3.96)$ & (5.51) & (1.23) & $(6.50)$ & $(8.01)$ & (1.87) & $(11.22)$ & (13.20) & (3.12) & (16.78) & (19.10) & $(4.51)$ \\
\hline & [37] & [27] & & [37] & [27] & & [37] & [27] & & [37] & [27] & \\
\hline \multirow[t]{3}{*}{ November } & 1.66 & 2.16 & -0.50 & 3.29 & 3.87 & -0.58 & 6.67 & 4.78 & 1.89 & 7.01 & 6.26 & 0.75 \\
\hline & (3.19) & (3.90) & (1.01) & (7.76) & (7.89) & (2.13) & $(11.22)$ & (13.87) & (3.54) & (15.26) & (16.81) & (4.34) \\
\hline & [46] & [18] & & [46] & [18] & & [46] & [18] & & [46] & [18] & \\
\hline \multirow[t]{3}{*}{ December } & 1.26 & 1.97 & -0.72 & 2.56 & 2.34 & 0.22 & 4.97 & 3.34 & 1.64 & 6.06 & 8.67 & -2.62 \\
\hline & (3.89) & (6.97) & $(1.92)$ & (7.06) & (10.84) & (3.02) & (9.74) & (20.02) & (5.46) & (14.94) & (20.96) & $(5.90)$ \\
\hline & [50] & [14] & & [50] & [14] & & [50] & [14] & & [50] & [14] & \\
\hline
\end{tabular}


For comparability with Cooper, McConnell and Ovtchinnikov (2006), we also report in Table 4 the results using the same sample period as theirs (January 1940 to December 2003) but for several holding periods besides 11 months. Consistent with them, a clear and significant pattern for the "other January" effect emerges: the spread between 11-month returns following positive and negative January returns are positive and statistically significant. However, there is nothing unique with their 11-month holding period as we find similar results when using 1-month, 3-, and 6-month holding period returns, all of which are statistically significant at the 5\% level. Nevertheless, none of the subsequent k-month return spread is statistically significant for conditioning months except for January.

Note that Cooper, McConnell and Ovtchinnikov's sample begins in 1940, although French's data provide monthly US stock returns since July 1926. Table 5 assembles our results for the alternative period of July 1926 to December 2003. While some evidence for "other January" effects are still present, but only at the weaker $10 \%$ level. In addition, there is evidence for a stronger and larger "other September" effect similar to our findings over the extended sample period (July 1926 to January 2012). Thus, the alleged "other January" effect seems to be specific to the period of January 1940 to December 2003 used in Cooper, McConnell and Ovtchinnikov (2006).

Following Farley, Hinich, and McGuire (1975) and Darrat, Li, Liu and Su (2011), we further inspect the sensitivity of the alleged anomaly to changes in time periods by dividing the sample at the midpoint to maximize the empirical power of our inspection. Table 6 displays the results for the first-half period (January 1927 to June 1963), while Table 7 does the same for the second-half period (July 1963 to January 2012).

In the first sub-period, the "other January" effect does not exist for any holding period. On average, the 3-month returns following positive February returns are lower than those following negative February returns. Compared to the results over the full period, we find a strong "other September" effect for all holding periods where returns in the next couple of months following positive September returns are larger than those following negative September returns.

The results for the latter sub-period also reject the "other January" effect at the 6month holding period, but support it at the 1-month holding period. The positive "other September" effect virtually disappears in this latter period. For other conditioning months, there are no effects for most of them, except for a positive "other April" effect for the 6month holding period, and a negative "other August" effect for the 6-month holding period". Again, these results persist in suggesting that the alleged "other January" effect is sample specific, lacking robustness across time and across holding periods.

\footnotetext{
${ }^{3}$ Since our expanded sample includes the recent and ongoing global finance crisis, one might raise concern that our results could be driven by this important even. Therefore, we reapplied our tests over the pre-crisis period of July 1926 through December 2006. The results, available upon request, are qualitatively similar to those obtained from the full period, implying that the global financial crisis is unlikely the culprit behind our results.
} 
Table 5: Testing the “Other Month" Anomalies (1926 July - December 2003)

\begin{tabular}{|c|c|c|c|c|c|c|c|c|c|c|c|c|}
\hline & \multicolumn{3}{|c|}{1} & \multicolumn{3}{|c|}{3} & \multicolumn{3}{|c|}{6} & \multicolumn{3}{|c|}{11} \\
\hline & + & - & Diff & + & - & Diff & + & - & Diff & + & - & Diff \\
\hline \multirow[t]{3}{*}{ January } & 0.75 & -0.47 & 1.22 & 2.72 & -1.00 & $3.73 * *$ & 6.24 & -1.23 & $7.47 * *$ & 9.32 & 1.72 & $7.60^{*}$ \\
\hline & $(4.42)$ & (3.25) & $(0.87)$ & (7.16) & (7.61) & $(1.75)$ & (11.01) & (12.68) & $(2.83)$ & (19.61) & (19.09) & (4.54) \\
\hline & [49] & [28] & & [49] & [28] & & [49] & [28] & & [49] & [28] & \\
\hline \multirow[t]{3}{*}{ February } & 0.24 & 0.34 & -0.10 & -0.42 & 4.30 & -4.71 & 2.42 & 7.12 & -4.70 & 5.89 & 11.13 & -5.24 \\
\hline & (5.41) & (4.58) & (1.14) & (10.98) & (14.87) & (3.06) & (9.41) & (20.65) & $(3.75)$ & (18.46) & $(26.60)$ & $(5.26)$ \\
\hline & [43] & [34] & & [43] & [34] & & [43] & [34] & & [43] & [34] & \\
\hline \multirow[t]{3}{*}{ March } & 1.71 & -0.41 & 2.12 & 3.79 & -0.33 & 4.12 & 3.46 & 2.68 & 0.79 & 8.08 & 8.72 & -0.64 \\
\hline & (6.60) & (6.71) & (1.58) & (14.61) & (13.73) & (3.31) & (15.49) & (19.12) & $(4.09)$ & (21.54) & $(25.96)$ & $(5.65)$ \\
\hline & [48] & [29] & & [48] & [29] & & [48] & [29] & & [48] & [29] & \\
\hline \multirow[t]{3}{*}{ April } & 0.92 & -0.95 & 1.87 & 2.99 & 0.66 & 2.33 & 3.85 & -1.49 & $5.34 *$ & 10.70 & 2.29 & $8.41 *$ \\
\hline & (5.64) & (6.31) & (1.44) & (8.61) & (8.95) & (2.05) & (11.54) & (15.37) & $(3.20)$ & (17.19) & (24.44) & $(5.04)$ \\
\hline & [47] & [30] & & [47] & [30] & & [47] & [30] & & [47] & [30] & \\
\hline \multirow[t]{3}{*}{ May } & 0.46 & 1.31 & -0.85 & 2.07 & 5.04 & -2.97 & 4.28 & 1.12 & 3.16 & 9.39 & 6.35 & 3.04 \\
\hline & (3.83) & $(7.20)$ & (1.38) & (6.96) & (17.86) & (3.34) & (12.33) & (17.41) & $(3.60)$ & (17.92) & (25.82) & $(5.30)$ \\
\hline & [47] & [30] & & [47] & [30] & & [47] & [30] & & [47] & [30] & \\
\hline \multirow[t]{3}{*}{ June } & 0.51 & 2.06 & -1.55 & 1.01 & 1.49 & -0.48 & 4.36 & 3.53 & 0.83 & 8.72 & 7.52 & 1.20 \\
\hline & (4.73) & (7.34) & (1.44) & (8.79) & (17.29) & (3.23) & (14.28) & (17.05) & $(3.62)$ & (20.67) & $(29.50)$ & $(5.90)$ \\
\hline & [42] & [35] & & {$[42]$} & [35] & & [42] & [35] & & [42] & [35] & \\
\hline \multirow[t]{3}{*}{ July } & 1.58 & 0.48 & 1.10 & 0.11 & -0.94 & 1.05 & 4.40 & 4.05 & 0.35 & 8.54 & 6.39 & 2.15 \\
\hline & $(6.35)$ & (6.29) & (1.40) & (9.43) & $(9.31)$ & (2.09) & (14.75) & (13.86) & (3.18) & (22.83) & $(22.90)$ & (5.17) \\
\hline & [44] & [34] & & [44] & [34] & & [44] & [34] & & [44] & [34] & \\
\hline \multirow[t]{3}{*}{ August } & -1.24 & -1.29 & 0.05 & -0.30 & 0.68 & -0.98 & 3.77 & 3.14 & 0.63 & 7.57 & 6.42 & 1.16 \\
\hline & (5.85) & (6.19) & (1.40) & (12.25) & (8.69) & (2.40) & (15.12) & (12.72) & $(3.22)$ & $(21.01)$ & (18.15) & (4.49) \\
\hline & [48] & [30] & & [48] & [30] & & [48] & [30] & & [48] & [30] & \\
\hline \multirow[t]{3}{*}{ September } & 1.26 & -1.03 & $2.29 *$ & 5.87 & 0.51 & $5.36 * *$ & 8.87 & 2.49 & $6.39 * *$ & 11.78 & 7.60 & 4.18 \\
\hline & (3.94) & (7.17) & (1.26) & $(6.90)$ & (10.42) & (1.96) & (11.89) & $(15.50)$ & (3.10) & (19.14) & (18.97) & (4.34) \\
\hline & [33] & [45] & & [33] & [45] & & [33] & [45] & & [33] & [45] & \\
\hline \multirow[t]{3}{*}{ October } & 1.66 & 0.83 & 0.83 & 4.14 & 4.62 & -0.48 & 5.46 & 6.71 & -1.25 & 7.68 & 8.93 & -1.25 \\
\hline & (4.44) & (6.13) & $(1.22)$ & $(8.05)$ & $(8.86)$ & (1.91) & (13.32) & (13.33) & $(3.00)$ & (17.32) & (22.04) & (4.49) \\
\hline & [42] & [36] & & [42] & [36] & & [42] & [36] & & [42] & [36] & \\
\hline \multirow[t]{3}{*}{ November } & 1.68 & 1.06 & 0.62 & 3.58 & 2.91 & 0.67 & 6.56 & 1.88 & 4.67 & 7.49 & 4.18 & 3.30 \\
\hline & (3.02) & (5.10) & (1.09) & (7.61) & (7.89) & (1.87) & (10.76) & (20.62) & $(4.24)$ & (16.22) & (20.33) & $(4.53)$ \\
\hline & [53] & [25] & & [53] & [25] & & [53] & [25] & & [53] & [25] & \\
\hline \multirow[t]{3}{*}{ December } & 1.39 & 1.95 & -0.56 & 2.46 & 0.98 & 1.48 & 5.19 & 0.85 & 4.35 & 6.65 & 5.55 & 1.10 \\
\hline & (4.15) & (6.42) & (1.64) & $(7.50)$ & (11.44) & $(2.87)$ & (12.81) & (21.09) & $(5.35)$ & (18.08) & (22.78) & $(5.95)$ \\
\hline & [61] & [17] & & [61] & [17] & & [61] & [17] & & [61] & [17] & \\
\hline
\end{tabular}

Notes: See notes to Table 2. 
Table 6: Testing the "Other Month" Anomalies (Midpoint Break: July 1926 - June 1963)

\begin{tabular}{|c|c|c|c|c|c|c|c|c|c|c|c|c|}
\hline & \multicolumn{3}{|c|}{1} & \multicolumn{3}{|c|}{3} & \multicolumn{3}{|c|}{6} & \multicolumn{3}{|c|}{11} \\
\hline & + & - & Diff & + & - & Diff & + & - & Diff & + & - & Diff \\
\hline \multirow[t]{3}{*}{ January } & 0.45 & 0.71 & -0.26 & 2.12 & -0.40 & 2.53 & 7.08 & 2.71 & 4.37 & 10.05 & 8.13 & 1.92 \\
\hline & $(4.95)$ & (2.98) & $(1.26)$ & (7.88) & (9.49) & $(3.00)$ & (13.38) & (12.39) & $(4.29)$ & (25.87) & (19.13) & (7.42) \\
\hline & [24] & [13] & & [24] & [13] & & [24] & [13] & & [24] & [13] & \\
\hline \multirow{2}{*}{ February } & (7.14) & (4.16) & (1.85) & (14.01) & (19.61) & (5.69) & (10.97) & (24.89) & (6.38) & $(24.21)$ & (32.08) & $(9.22)$ \\
\hline & [21] & [16] & & [21] & [16] & & [21] & [16] & & [21] & [16] & \\
\hline \multirow[t]{3}{*}{ March } & 2.26 & -1.03 & 3.29 & 5.26 & 0.14 & 5.12 & 6.16 & 6.03 & 0.12 & 12.87 & 10.21 & 2.66 \\
\hline & $(8.81)$ & $(8.22)$ & $(2.81)$ & (20.45) & (16.82) & (6.07) & (18.84) & (21.87) & (6.60) & (26.41) & (32.66) & (9.83) \\
\hline & [22] & [15] & & [22] & [15] & & [22] & [15] & & [22] & [15] & \\
\hline \multirow{2}{*}{ April } & (7.69) & (7.61) & (2.56) & $(9.30)$ & (9.90) & (3.18) & (13.50) & (18.81) & (5.35) & (20.47) & (29.14) & (8.26) \\
\hline & [21] & [16] & & [21] & [16] & & [21] & [16] & & [21] & [16] & \\
\hline \multirow[t]{3}{*}{ May } & 0.98 & 2.55 & -1.57 & 3.52 & 12.78 & -9.25 & 7.50 & 2.49 & 5.02 & 15.22 & 4.69 & 10.53 \\
\hline & (4.49) & (9.99) & (2.74) & (6.80) & (23.36) & (6.30) & (12.92) & (24.07) & $(6.92)$ & (19.15) & (35.17) & (10.02) \\
\hline & [24] & [13] & & [24] & [13] & & [24] & [13] & & [24] & [13] & \\
\hline \multirow[t]{3}{*}{ June } & 1.27 & 5.01 & -3.73 & 1.84 & 5.88 & -4.05 & 6.50 & 6.03 & 0.46 & 11.68 & 10.54 & 1.14 \\
\hline & (5.46) & (8.69) & (2.46) & (10.33) & (21.77) & (5.85) & (17.82) & $(21.01)$ & (6.44) & $(26.28)$ & (38.45) & (11.01) \\
\hline & [21] & [16] & & [21] & [16] & & [21] & [16] & & [21] & [16] & \\
\hline \multirow[t]{2}{*}{ July } & 1.77 & 2.68 & -0.90 & 0.43 & -2.88 & 3.31 & 5.13 & 3.03 & 2.10 & 10.44 & 4.32 & 6.12 \\
\hline & $(7.70)$ & (5.11) & $(2.12)$ & (10.45) & (11.79) & $(4.12)$ & (16.67) & (20.26) & $(7.01)$ & (26.83) & (30.29) & (10.85) \\
\hline \multirow[t]{3}{*}{ August } & -1.61 & -1.64 & 0.02 & -0.26 & -1.59 & 1.33 & 4.91 & -0.56 & 5.47 & 10.52 & 4.92 & 5.60 \\
\hline & $(7.35)$ & $(7.62)$ & (2.59) & (13.80) & (11.68) & $(4.31)$ & (17.68) & (14.35) & (5.42) & (24.14) & $(17.80)$ & (6.98) \\
\hline & [25] & [12] & & [25] & [12] & & [25] & [12] & & [25] & [12] & \\
\hline \multirow[t]{3}{*}{ September } & 1.95 & -2.81 & $4.76^{* * *}$ & 7.83 & -2.11 & $9.95 * *$ & 12.43 & -1.89 & $14.32 * *$ & 19.21 & 5.63 & $13.57 * *$ \\
\hline & (3.90) & (6.58) & $(1.72)$ & (5.69) & (11.83) & $(3.00)$ & (10.85) & (15.28) & $(4.23)$ & (15.55) & (19.65) & (5.67) \\
\hline & [17] & [20] & & [17] & [20] & & [17] & [20] & & [17] & [20] & \\
\hline \multirow[t]{3}{*}{ October } & 1.05 & 1.50 & -0.45 & 3.45 & 5.44 & -1.98 & 4.09 & 7.93 & -3.84 & 8.77 & 12.91 & -4.14 \\
\hline & $(5.03)$ & (6.58) & (1.86) & (10.26) & (9.55) & (3.16) & (16.75) & (13.62) & (4.93) & (19.31) & (22.43) & (6.69) \\
\hline & [17] & [20] & & [17] & [20] & & [17] & [20] & & [17] & [20] & \\
\hline \multirow[t]{3}{*}{ November } & 2.57 & -0.32 & $2.89^{*}$ & 5.52 & -0.36 & $5.89 * *$ & 8.90 & -2.44 & 11.34 & 11.42 & 3.50 & 7.91 \\
\hline & $(2.36)$ & (5.95) & (1.71) & (6.46) & (7.04) & $(2.33)$ & (10.89) & $(25.50)$ & $(7.22)$ & (18.26) & (22.46) & (7.20) \\
\hline & [25] & [12] & & [25] & [12] & & [25] & [12] & & [25] & [12] & \\
\hline \multirow[t]{3}{*}{ December } & 1.35 & 1.75 & -0.40 & 2.76 & -1.41 & 4.17 & 6.64 & -3.59 & 10.24 & 9.05 & 6.77 & 2.28 \\
\hline & (4.46) & (3.70) & $(1.52)$ & (7.59) & (11.00) & $(4.02)$ & (15.09) & (22.18) & (8.36) & (20.11) & (29.13) & (10.99) \\
\hline & [30] & [7] & & [30] & [7] & & [30] & [7] & & [30] & [7] & \\
\hline
\end{tabular}

Notes: See notes to Table 2. 
Table 7: Testing the “Other Month" Anomalies (Midpoint Break: July 1963 - January 2012)

\begin{tabular}{|c|c|c|c|c|c|c|c|c|c|c|c|c|}
\hline & \multicolumn{3}{|c|}{1} & \multicolumn{3}{|c|}{3} & \multicolumn{3}{|c|}{6} & \multicolumn{3}{|c|}{11} \\
\hline & + & - & Diff & + & - & Diff & + & - & Diff & + & - & Diff \\
\hline \multirow[t]{3}{*}{ January } & 1.00 & -1.54 & $2.53^{* * *}$ & 3.17 & -0.17 & $3.34 *$ & 4.56 & -2.08 & $6.64 * *$ & 7.95 & -1.09 & 9.04* \\
\hline & (3.73) & (3.79) & $(1.08)$ & (6.16) & $(6.42)$ & $(1.85)$ & (8.06) & (13.11) & (3.28) & (10.71) & (21.46) & (5.12) \\
\hline & [29] & [19] & & [29] & [19] & & [29] & [19] & & [29] & [19] & \\
\hline \multirow{2}{*}{ February } & $(2.46)$ & $(4.68)$ & (1.07) & (5.84) & $(9.52)$ & (2.32) & $(7.62)$ & $(15.86)$ & (3.65) & (10.63) & (22.04) & (4.96) \\
\hline & [26] & [22] & & [26] & [22] & & [26] & [22] & & [26] & [22] & \\
\hline \multirow[t]{3}{*}{ March } & 1.71 & 0.20 & 1.51 & 2.41 & -0.62 & 3.04 & 1.88 & -1.16 & 3.04 & 5.56 & 4.34 & 1.22 \\
\hline & (4.09) & $(4.62)$ & $(1.32)$ & (7.40) & $(9.09)$ & (2.51) & (13.17) & (14.47) & $(4.13)$ & (16.79) & (20.06) & (5.66) \\
\hline & [31] & [17] & & [31] & [17] & & [31] & [17] & & [30] & [17] & \\
\hline \multirow{2}{*}{ April } & (3.65) & (4.38) & (1.24) & $(7.76)$ & $(7.30)$ & $(2.21)$ & (11.11) & (9.64) & (3.02) & (16.88) & (17.61) & (5.30) \\
\hline & [32] & [16] & & [32] & [16] & & [32] & [16] & & [31] & [16] & \\
\hline \multirow[t]{3}{*}{ May } & -0.32 & -0.07 & -0.25 & 0.48 & -1.32 & 1.79 & 0.38 & 0.60 & -0.22 & 2.82 & 9.09 & -6.27 \\
\hline & $(3.16)$ & $(4.00)$ & (1.06) & (6.95) & $(8.63)$ & (2.26) & (13.13) & (10.38) & (3.30) & (16.66) & (16.49) & (4.84) \\
\hline & [28] & [20] & & [28] & [20] & & [28] & [20] & & [28] & [19] & \\
\hline \multirow[t]{3}{*}{ June } & -0.22 & -0.02 & -0.20 & 0.25 & -1.43 & 1.69 & 2.62 & 1.74 & 0.88 & 5.86 & 5.69 & 0.16 \\
\hline & $(3.86)$ & (4.94) & (1.24) & $(6.81)$ & (11.77) & (2.66) & $(9.19)$ & (14.96) & (3.47) & (12.33) & $(20.51)$ & (4.77) \\
\hline & [23] & [25] & & [23] & [25] & & [23] & [25] & & [23] & [24] & \\
\hline \multirow[t]{2}{*}{ July } & 0.92 & -0.32 & 1.24 & 0.19 & -0.60 & 0.79 & 4.40 & 3.01 & 1.39 & 6.15 & 5.84 & 0.31 \\
\hline & $(3.05)$ & $(6.10)$ & (1.27) & (7.33) & $(9.28)$ & (2.34) & (10.76) & (12.93) & (3.35) & (12.95) & (20.19) & (4.68) \\
\hline \multirow[t]{3}{*}{ August } & -0.61 & -0.83 & 0.21 & -0.62 & 2.73 & -3.35 & 1.26 & 6.78 & -5.52 & 3.73 & 8.11 & -4.38 \\
\hline & $(4.07)$ & $(5.62)$ & (1.38) & (11.77) & $(6.01)$ & (2.54) & (14.31) & (11.87) & (3.72) & (16.62) & (18.31) & (5.06) \\
\hline & [28] & [21] & & [28] & [21] & & [28] & [20] & & [28] & [20] & \\
\hline \multirow[t]{3}{*}{ September } & 0.66 & 0.11 & 0.54 & 4.15 & 1.95 & 2.20 & 5.41 & 4.55 & 0.86 & 3.83 & 8.46 & -4.63 \\
\hline & (3.64) & (8.33) & (1.74) & (7.04) & (10.09) & (2.35) & (11.48) & (16.48) & (3.97) & (17.47) & (18.65) & (5.11) \\
\hline & [22] & [27] & & [22] & [27] & & [22] & [26] & & [22] & [26] & \\
\hline \multirow[t]{3}{*}{ October } & 1.77 & 0.04 & 1.74 & 4.24 & 2.95 & 1.29 & 6.05 & 5.47 & 0.57 & 5.69 & 5.45 & 0.24 \\
\hline & (3.98) & $(5.74)$ & (1.46) & (6.58) & $(8.50)$ & $(2.24)$ & (10.64) & (12.79) & (3.46) & (16.33) & (19.70) & (5.32) \\
\hline & [30] & [19] & & [30] & [19] & & [29] & [19] & & [29] & [19] & \\
\hline \multirow[t]{3}{*}{ November } & 1.16 & 2.01 & -0.85 & 2.24 & 3.50 & -1.27 & 4.68 & 5.44 & -0.76 & 4.63 & 3.32 & 1.31 \\
\hline & $(3.29)$ & $(3.66)$ & (1.05) & (7.82) & (9.64) & (2.76) & $(9.78)$ & (13.96) & (3.79) & (12.60) & (21.29) & (5.61) \\
\hline & [33] & [16] & & [33] & {$[15]$} & & [33] & [15] & & [33] & [15] & \\
\hline \multirow[t]{3}{*}{ December } & 1.12 & 1.32 & -0.20 & 1.96 & 1.52 & 0.44 & 3.53 & 2.59 & 0.94 & 5.12 & 0.53 & 4.59 \\
\hline & $(4.03)$ & $(8.03)$ & (2.45) & (7.27) & (12.00) & $(3.70)$ & $(9.49)$ & (20.34) & (6.21) & (15.08) & (22.61) & (7.09) \\
\hline & [38] & [11] & & [37] & [11] & & [37] & [11] & & [37] & [11] & \\
\hline
\end{tabular}


Next, we investigate whether the "other January" anomaly is due to momentum or contrarian strategies. Consider for example the month of January 2011. If stock prices fell from July to December 2010, fund managers may sell the losing stocks to realize capital gains, and buy them back some time later in 2011. Hence, the January and the subsequent 11 month returns would be negatively related to the July-December return in the previous year. This is the contrarian argument for the "other January" anomaly. However, the "other January" anomaly can also be related to the momentum strategy. If stock prices rise from July to December 2010, fund manager may continue to buy in 2011, in anticipation that prices will continue to rise. Thus, January and the subsequent 11-month returns would be positively related to the July-December return in the previous year.

Table 8 tests whether the "other January" anomaly is related to the contrarian or the momentum strategies. The table presents the regression results on the 11-month buy-and-hold returns from February to December against the returns in January, and also the stock returns from July to December from the previous year along with their interaction term. The coefficients for the January return and the interaction term are significantly positive for the sub-periods 1940-2003 and 1963-2011, but not for any other sub-periods. Thus, there is some evidence indicating that the "other January" effect may be related to the momentum strategy.

Table 8: Explaining the "Other January" Effect: Do Contrarian or Momentum Strategies Matter?

\begin{tabular}{crrrr}
\hline Regression & \multicolumn{4}{c}{$\mathbf{r 2} \mathbf{1 2}_{\mathbf{t}}=\mathbf{a}_{\mathbf{0}}+\mathbf{a}_{\mathbf{1}} * \mathbf{r} \mathbf{1}_{\mathbf{t}}+\mathbf{a}_{\mathbf{2}} * \mathbf{m o m}_{\mathbf{t}}+\mathbf{a}_{\mathbf{3}} *\left(\mathbf{r 1}_{\mathbf{t}} * \mathbf{m o m}_{\mathbf{t}}\right)+\mathbf{e}_{\mathbf{t}}$} \\
\hline \multirow{2}{*}{$1927-2011$} & $\mathbf{a}_{\mathbf{0}}$ & $\mathbf{a}_{1}$ & $\mathbf{a}_{\mathbf{2}}$ & $\mathbf{a}_{\mathbf{3}}$ \\
& $5.40^{* *}$ & 0.26 & 0.09 & $0.05^{* *}$ \\
& $(2.67)$ & $(0.55)$ & $(0.44)$ & $(2.03)$ \\
& & & & \\
$1940-2003$ & $5.97 * *$ & $0.99 * *$ & -0.13 & $0.05^{*}$ \\
& $(2.90)$ & $(3.07)$ & $(-0.86)$ & $(1.91)$ \\
& & & & \\
$1927-2003$ & $5.08^{* *}$ & 0.31 & 0.18 & 0.03 \\
& $(2.30)$ & $(0.64)$ & $(0.78)$ & $(1.17)$ \\
& & & & \\
$1927-1963$ & $8.05^{* *}$ & -0.83 & 0.23 & 0.05 \\
& $(2.77)$ & $(-0.79)$ & $(0.81)$ & $(1.36)$ \\
& & & & \\
$1964-2011$ & $4.02 * *$ & $0.87 * *$ & $-0.19 *$ & $0.07 * *$ \\
& $(2.04)$ & $(2.48)$ & $(-1.80)$ & $(3.01)$ \\
& & & & \\
\hline
\end{tabular}

Notes: See notes to Table 2. R2_12 is the holding period return from February to December. $\mathrm{r} 1$ is the January return. Mom is the holding period return from last July to last December. Newey-West adjusted t-statistics (with 4 lags) are in parentheses below the regression coefficients. $* *$ and $*$ denote statistical significance at the $5 \%$ and $10 \%$ levels, respectively. 
We also investigate in Table 9 if there is any time trend in the "other January" effect for the 1-, 3-, 6- and 11-month holding periods over the full sample. Regression 1 contains the results of the holding period returns against January return and its interaction term with a 1963 dummy variable (equals to 1 for the year 1963 and after and 0 otherwise). The results exhibit no statistical significance for the interaction term between January return and the post-1962 dummy for all holding periods. Regression 2 suggests only marginal significance for the interaction term between January return and the time trend variable. These results fail to support any significance for the presence time trends in the "other January" effect.

Table 9: Explaining the "Other January" Effect: Is There Any Time trend?

\begin{tabular}{|c|c|c|c|}
\hline Regression 1 & \multicolumn{3}{|c|}{${ }^{H P R} R_{t}=a_{0}+a_{1} * r 1_{t}+a_{2} *\left(r 1_{t} * \operatorname{dum} 1963_{t}\right)+e_{t}$} \\
\hline HPR1 & $\begin{array}{r}\mathbf{a}_{0} \\
0.04 \\
(0.12)\end{array}$ & $\begin{array}{r}\mathbf{a}_{1} \\
0.12 \\
(1.21)\end{array}$ & $\begin{array}{r}\mathbf{a}_{2} \\
0.07 \\
(0.51)\end{array}$ \\
\hline HPR3 & $\begin{array}{c}1.28^{*} \\
(1.67)\end{array}$ & $\begin{array}{r}0.24 \\
(0.85)\end{array}$ & $\begin{array}{r}0.01 \\
(0.02)\end{array}$ \\
\hline HPR6 & $\begin{array}{r}3.21 * * \\
(2.38)\end{array}$ & $\begin{array}{r}-0.03 \\
(-0.05)\end{array}$ & $\begin{array}{r}0.49 \\
(0.82)\end{array}$ \\
\hline HPR11 & $\begin{array}{c}6.49 * * \\
(3.54)\end{array}$ & $\begin{array}{r}-0.40 \\
(-0.36)\end{array}$ & $\begin{array}{r}0.83 \\
(0.71)\end{array}$ \\
\hline
\end{tabular}

\begin{tabular}{|c|c|c|c|}
\hline Regression 2 & \multicolumn{3}{|c|}{$\mathrm{HPR}_{t}=\mathrm{a}_{0}+\mathrm{a}_{1} * r 1_{t}+a_{2} *\left(r 1_{t} * \operatorname{trend} 1963_{t}\right)+e_{t}$} \\
\hline HPR1 & $\begin{array}{r}\mathbf{a}_{0} \\
0.07 \\
(0.19)\end{array}$ & $\begin{array}{r}\mathbf{a}_{1} \\
0.11 \\
(1.22)\end{array}$ & $\begin{array}{r}\mathbf{a}_{2} \\
0.00 \\
(0.61)\end{array}$ \\
\hline HPR3 & $\begin{array}{r}1.14 \\
(1.56)\end{array}$ & $\begin{array}{c}0.47 * \\
(1.87)\end{array}$ & $\begin{array}{l}-0.02 * \\
(-1.66)\end{array}$ \\
\hline HPR6 & $\begin{array}{r}3.08 * * \\
(2.24)\end{array}$ & $\begin{array}{r}0.39 \\
(0.77)\end{array}$ & $\begin{array}{r}-0.01 \\
(-0.31)\end{array}$ \\
\hline HPR11 & $\begin{array}{c}6.42 * * \\
(3.52)\end{array}$ & $\begin{array}{r}0.07 \\
(0.08)\end{array}$ & $\begin{array}{r}0.01 \\
(0.15)\end{array}$ \\
\hline
\end{tabular}

Notes: See notes to Tables 2 and 8. HPR denotes holding period return, (HPR1, HPR3, HPR6, HPR11) are for holding period of 1, 3, 6, and 11 months, respectively. Dum1963 is a dummy variable if the year is greater than or equal to 1963 . Trend1963 is a time-trend variable for the year 1963. 


\section{Conclusion}

This paper revisits Cooper, McConnell, and Ovtchinnikov's (2006) support for the "other January" anomaly in the US market whereby the 11-month holding period returns following positive January returns are on average higher than those 11 months following negative January returns. Under this scenario, January returns can predict the subsequent 11month holding period returns.

We re-examine this anomaly in the US stock market over the extended period from July 1926 to January 2012. Within the period of January 1940 to December 2003 originally used by Cooper, McConnell, and Ovtchinnikov, we find support for the alleged "other January" effect for the 11-month holding period as well as for other alternative holding periods (1, 3, and 6). However, this anomaly disappears in the expanded period of July 1926 to January 2012. The results from this expanded sample consistently suggest that the 11month returns following positive January returns are not statistically different from those following negative January returns. We find the 6-month stock returns following positive January returns are higher than those following negative January returns. This finding suggests that there is a momentum in the first 6 months which then reverts in the following 5 months. Moreover, this alleged anomaly is not unique with the month of January as similar and perhaps stronger results emerged for other months, particularly February and September. Our results cast further doubt on Cooper, McConnell, and Ovtchinnikov's "other January" effect and suggest that not only this alleged anomaly lacks support from non-US international markets as several recent studies suggest, but also that the alleged anomaly is sample-period sensitive and is not unique with the month of January.

\section{Acknowledgements}

Bin Li thanks Griffith Business School for financial support under the 2012 Griffith Business School Internal Researcher Grant. The authors wish to thank the Editor and two anonymous reviewers for several useful comments. The usual disclaimer applies. 


\section{References}

Bohl, MT and CA Salm (2010). The other January effect: International evidence. The European Journal of Finance, 16, 173-182.

Brusa, J and P Liu (2004). The day-of-the-week and the week-of-the-month effects: An analysis of investors' trading activities. Review of Quantitative Finance and Accounting, 23, 19-30.

Cooper, MJ, JJ McConnell and AV Ovtchinnikov (2006). The other January effect. Journal of Financial Economics, 82, 315-341.

Cooper, MJ, JJ McConnell and AV Ovtchinnikov (2009). What's the best way to trade using the January barometer?. SSRN Working Paper.

Darrat AF, B Li, B Liu and JJ Su (2011). A fresh look at seasonal anomalies: An international perspective. International Journal of Business and Economics, 10, 93-116.

Dzhabarov, C and WT Ziemba (2010). Do seasonal anomalies still work?. Journal of Portfolio Management, 36, 93-104.

Easterday, KE, PK Sen and JA Stephan (2009). The persistence of the small firm/January effect: Is it consistent with investors' learning and arbitrage efforts?. The Quarterly Review of Economics and Finance, 49, 1172-1193.

Easton, SA and SM Pinder (2007). A refutation of the existence of the other January effect. International Review of Finance, 7, 89-104.

Fama, E (1970). Efficient capital markets: A review of theory and empirical work. Journal of Finance, 25, 383-417.

Farley, JU, MJ Hinich and TW McGuire (1975). Some comparisons of tests for a shift in the slopes of a multivariate linear time series model. Journal of Econometrics, 3, 297-318.

$\mathrm{Gu}, \mathrm{A}$ (2004). The reversing weekend effect: Evidence from the US equity markets. Review of Quantitative Finance and Accounting, 22, 5-14.

Kunkel, RA, WS Compton and S Beyer (2003). The turn-of-the-month effect still lives: The international evidence. International Review of Financial Analysis, 12, 207-221.

Marshall, BR and N Visaltanachoti (2010). The other January effect: Evidence against market efficiency. Journal of Banking and Finance, 34, 2413-2424.

Russolillo, S (2010). 'Santa Claus Rally' at top of investors' wish list. Wall Street Journal, November 29.

Stivers, C, L Sun and Y Sun (2009). The other January effect: International, style, and sub period evidence. Journal of Financial Markets, 12, 521-546.

Sturm, RR (2009). The 'other' January effect and the presidential election cycle. Applied Financial Economics, 19, 1355-1363.

Sun, Q and WHS Tong (2002). Another new look at the Monday effect. Journal of Business Finance and Accounting, 29, 1123-1147.

Tonchev, D and TH Kim (2004). Calendar effects in Eastern European financial markets: Evidence from the Czech Republic, Slovakia and Slovenia. Applied Financial Economics, 14, 1035-1043.

Tu, A (2003). The shift of weekend effects in Taiwan's equity index return: Index futures listings or other alternative explanations. Review of Pacific Basin Financial Markets \& Policies, 6, 549-572. 